\title{
Ultrasound Simulation-How to Adapt It for Donald School Needs?
}

Sanja Kupesic Plavsic

\begin{abstract}
"Tell me, and I will forget. Show me, and I may remember. Involve me, and I will understand."
\end{abstract}

-Confucius, $450 \mathrm{BC}$

Use of simulation technology allows learners to perform repetitive ultrasound examinations and practice at their own pace without compromising patient comfort and/or safety. Structured training and incremental exposure to different pathologies enables gradual knowledge and scanning skill acquisition. Ultrasound simulation helps shorten novices' learning curve in image orientation, hand-eye coordination, interpretation skills and clinical reasoning. Learning new scanning protocols in a scaled, self-directed manner is very useful, especially when considering the patient discomfort, intimate nature of OB GYN sonography and limited faculty spare time. New generation of ultrasound simulators provides an opportunity for longitudinal monitoring of the learners' scanning skills based on the outcomes obtained by validated tools for assessment. More complex modules can be used for advanced Donald School courses under the guidance of experts to assess skill levels and suggest interventions to improve the quality and timeliness of performing comprehensive examination.

Keywords: Doppler, Pelvic ultrasound, Psychomotor, Virtual reality ultrasound simulators.

How to cite this article: Plavsic SK. Ultrasound SimulationHow to Adapt It for Donald School Needs? Donald School J Ultrasound Obstet Gynecol 2017;11(3):179-183.

Source of support: Nil

Conflict of interest: None

\section{INTRODUCTION}

Ultrasound (US) is the most commonly used diagnostic tool for the evaluation of female pelvic organs, infertility, early pregnancy, and prenatal assessment. Pronounced as "the stethoscope of the future," US was introduced

Professor and Associate Dean

Department of Obstetrics and Gynecology, Center for Advanced Teaching and Assessment in Clinical Simulation; Paul L. Foster School of Medicine, Texas Tech University Health Sciences EI Paso, EI Paso, Texas, USA

Corresponding Author: Sanja Kupesic Plavsic, Professor and Associate Dean, Department of Obstetrics and Gynecology Center for Advanced Teaching and Assessment in Clinical Simulation; Paul L. Foster School of Medicine, Texas Tech University Health Sciences EI Paso, El Paso, Texas, USA e-mail: sanja.kupesic@ttuhsc.edu into the busy clinical environments of obstetricians and gynecologists; family medicine, internal medicine, and emergency medicine physicians; anesthesiologists; cardiologists; trauma surgeons; pediatricians; and neonatologists. Bedside US has become a reality, and during the last decade over 50 models of portable US scanners (also referred as laptop, handheld, and cart-based units) from 20 manufacturers became commercially available. ${ }^{1}$ These high-resolution devices have operational modes that are comparable with full-size scanners including B-mode, M-mode, tissue harmonic imaging, color and power Doppler, continuous and pulsed wave Doppler, and more recently three-dimensional (3D) and fourdimensional imaging. However, the widespread use of US in clinical practice could not have been introduced without dedicated and competent educators and proctors who successfully transfer their psychomotor and cognitive skills in image acquisition and interpretation to their learners.

In this editorial, I will focus on standards of US training and difference between traditional and simulation training models. I will review different US simulators available in the field of obstetrics and gynecology, and discuss how they can be implemented in Ian Donald educational programs.

\section{US Training Models: Traditional vs Simulation}

Traditional US training model based on "see one, do one, teach one" strategy requires a patient with certain pathologic condition, an US machine, a qualified instructor, and a trainee. It may take months and/or years to scan a sufficient number of pathologic cases to develop US competency in a certain discipline and/or area. Patients with severe pain and anxiety may complain of the duration of the examination, especially when scans are performed transvaginally, transrectally, or transesophageally. Furthermore, long exposure to the sound waves (e.g., prolonged Doppler examination of ductus venosus and fetal heart in early pregnancy) increases the risk of thermal effect on a vulnerable fetal tissue. Similarly, Doppler studies of the middle cerebral artery in late pregnancy raise the concern of the heating effect, caused by high absorption of the energy in already developed bone tissue, and reflection at bone-soft tissue interfaces. Precautionary use of a limited time for an US and/or Doppler scan clearly inhibits the trainee to spend sufficient time for an obstetric scan. 
Recent technological advances have provided the tools to improve traditional US training and assessment strategies to achieve mastery in different US techniques in a more consistent way and in a shorter period of time. High-end US simulators provide learners (students, residents, fellows, etc.), practicing physicians, sonographers, nurses, and other health care providers with an opportunity to acquire and improve their sonographyrelated diagnostic and interventional skills on a variety of virtual cases. Patients in teaching hospitals are commonly confronted with extended examination times and repeated procedures performed by inexperienced trainees. Development of US simulation enables creation and delivery of well-designed, learner-centered, performance-based curriculum placed in a safe educational environment, on a bridge between classroom learning and real-life clinical experience. Realistic US models and scalable modules with varying degrees of difficulty allow the learners to apply the theoretical knowledge and clinical reasoning in a controlled setting away from the patients, ultimately improving patient safety.

While performing an US examination, competent practitioners use their psychomotor skills to scan the area of interest, integrate the information from patient history and clinical findings, interpret the images, and after appropriate clinical decision-making, communicate the information efficiently and professionally to the patient, and finally, write a well-structured report. Improvement in technology resulted in creation of realistic and complex US simulation modules that can mimic the entire process of a comprehensive US examination. Systematic use of clinical simulation can improve patient safety and overcome the challenges associated with cost-effective delivery of medical and health care education in busy clinical departments and/or US centers.

Image orientation and hand-eye coordination happen at different speeds for different learners, especially in the early stages of US training. Use of simulation technology allows learners to perform repetitive examinations and practice at their own pace without compromising patient comfort and/or safety. Structured training and incremental exposure to different pathologies enables gradual knowledge and scanning skill acquisition. Ability to systematically examine the anatomical structures, discuss the sonographic findings with proctors and peers, and focus and obtain feedback about the scanning technique without interaction with real patients is particularly beneficial in the introductory phase of transvaginal US education. Timely and specific feedback and ability to discuss technical aspects of scanning improves knowledge integration and increases the confidence of the individual learner. More advanced US simulators compare and annotate US and anatomical landmarks,

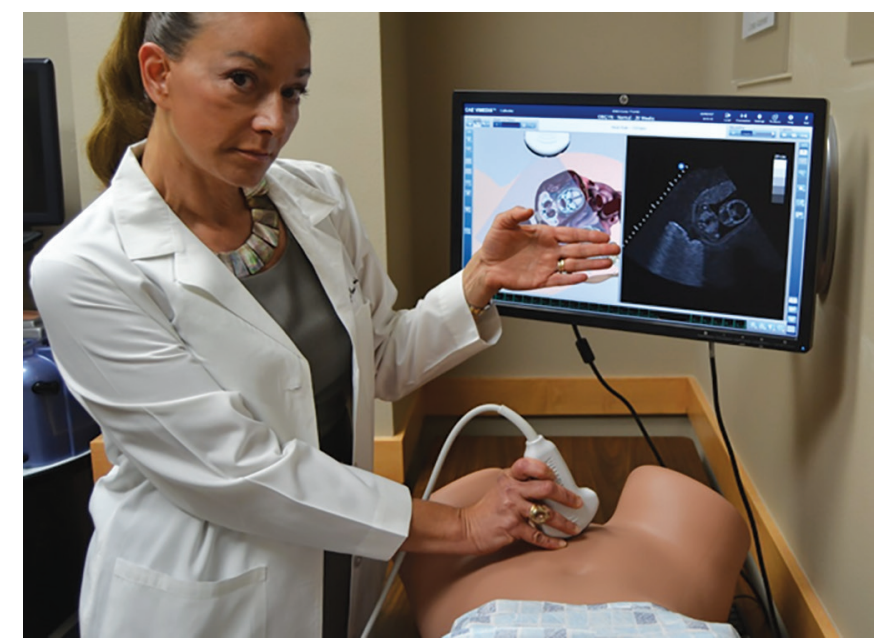

Fig. 1: Dr Kupesic performs OB US simulation on Vimedix US simulator, featuring second-trimester scanning with realistic fetal and maternal anatomy. Augmented reality display on the left side of the screen includes interactive animated 3D anatomical depiction of organs, structures, and/or abnormalities

which accelerates and improves self-directed learning and assessment (Fig. 1).

"What came first, the chicken or the egg?" is a question that has vexed philosophers since the ancient Greeks. Using the same question metaphorically, and in the context of this editorial, I'll ask a rhetorical question: "What came first, the theoretical knowledge or the art of scanning?" I believe that exposure to US simulation experience early in the curriculum maximizes the efficiency of sonography training. Gradual exposure to more demanding and challenging cases enables strategic development of a truly integrated curriculum, application of adequate training methodology, and continuous monitoring of the adequacy of the learning objectives and needs assessment in relation to the learner's level of competency.

\section{Standards of US Training}

Despite advancement in technology, US remains highly operator-dependent and therefore must be provided by properly trained and committed practitioners using the appropriate high-resolution US equipment. Given the variability among and between trainees in scanning, hand-eye coordination and interpretation skills, it is unlikely that standardization can be done based on setting a minimum number of scans to reflect candidates' skills. There is a lack of standardization to assess whether health care practitioners are able to provide a safe and effective US service relevant to their clinical practice nationwide, as well as lack of standardization for US training among different institutions and countries.

According to the American Institute of Ultrasound in Medicine guidelines and standards for US training, Obstetrics and Gynecology (OB GYN) residents and/or 
fellows are required to document minimum of 3 months of US training (equivalent to 300 US examinations), before independently performing and interpreting female pelvic US. ${ }^{2}$ On the contrary, the International Society of Ultrasound in Obstetrics and Gynecology suggested a minimum of $200 \mathrm{OB}$ scans to be performed by residents in OB GYN. ${ }^{3,4}$ However, the number of scans or procedures performed by trainees is commonly influenced by duty hour restrictions and reduced exposure time to US scanning. ${ }^{5,6}$ In order to maximize the learning efficiency within precious training duty hours, achieve the highest possible performance level, and improve patient safety, educators should consider conducting simulation in a safe learning environment, especially in early stages of US training.

The aim of Ian Donald Inter-University School of Medical Ultrasound is to promote education, research, and art of US scanning, and to maintain high standards of competency. Our meetings and conferences present the latest evidence-based education in the field of OB GYN US that helps our participants to significantly improve their diagnostic and clinical accuracy. Donald School basic and advanced courses are designed by regional school directors, experienced clinicians, sonographers, and other imaging professionals with the aim to provide highquality educational opportunities specific to their learners' needs assessment to advance their knowledge and skills in OB GYN US scanning. ${ }^{7}$ Following completion of Ian Donald School basic US courses, participants are able to perform common examinations safely and accurately, recognize and differentiate normal anatomy and pathology, diagnose common abnormalities within certain organ/ systems, and understand the relationship between US imaging and other diagnostic and/or imaging techniques. Following completion of the advanced courses, learners are able to recognize and correctly diagnose almost all conditions within the relevant organ/system, perform US-guided procedures, conduct US-related research, and teach US to trainees and basic-course participants. Learners who have successfully completed a comprehensive Donald School postgraduate course are able to perform specialized US examinations and advanced US-guided invasive procedures, teach US at all levels, conduct substantial research in US, and pursue further technological developments in the field of sonography. Following training and competency assessment, Donald School course graduates are obliged to regular continuing professional development and keeping up to date with the relevant literature. It is individual responsibility of each graduate to ensure that his/her practical skills are maintained by ensuring the relevant continuous US practice.

\section{Models of US Simulation}

High-fidelity US simulators are composed of a human mannequin, a mock probe, and a computer. Typically, the mock probe contains a 3D sensor, capable of acquiring virtual position data instantaneously. The probe is connected directly to the computer, whose screen displays the US image depending upon the probe's position and movements. ${ }^{8}$ Information about the location and position of the mock probe on the mannequin is transferred to the computer and displayed as related two-dimensional (2D) images. ${ }^{9}$ A haptic device can be used instead of a mannequin, allowing measurement of the pressure applied to the probe and providing realistic feedback on this force ${ }^{10}$ Limitations of the latter model are absence of a mannequin and lower range of movements to the probe.

Most virtual reality US simulators provide a 3D animated illustration of the anatomy surrounding the probe, marking its position and relationship with different organs and systems. ${ }^{10}$ This option improves hand-eye coordination of the learners and is particularly useful in the initial phase of US training (Fig. 2). Animated illustrations of the region/area are optional and can be hidden on demand in later stages of training and/or during assessment.

Based on method of image generation, US simulators are divided into four main groups:

1. Online simulation: Web-based programs that use mouse-operated controls to change scan planes and simulate probe manipulation, and display US images corresponding to the particular scan plane (e.g., SonoSim US Training Solution).

2. Phantom-based simulators: Use of simple technology, such as plastic fetal phantom scanned with a regular US machine (e.g., "Space Fan ST" by Kyoto Kagaku Company used for second-trimester scan simulation).

3. Interpolative model-based simulators: 2D US images in interpolative model-based simulator generated from 3D volumes previously acquired during actual

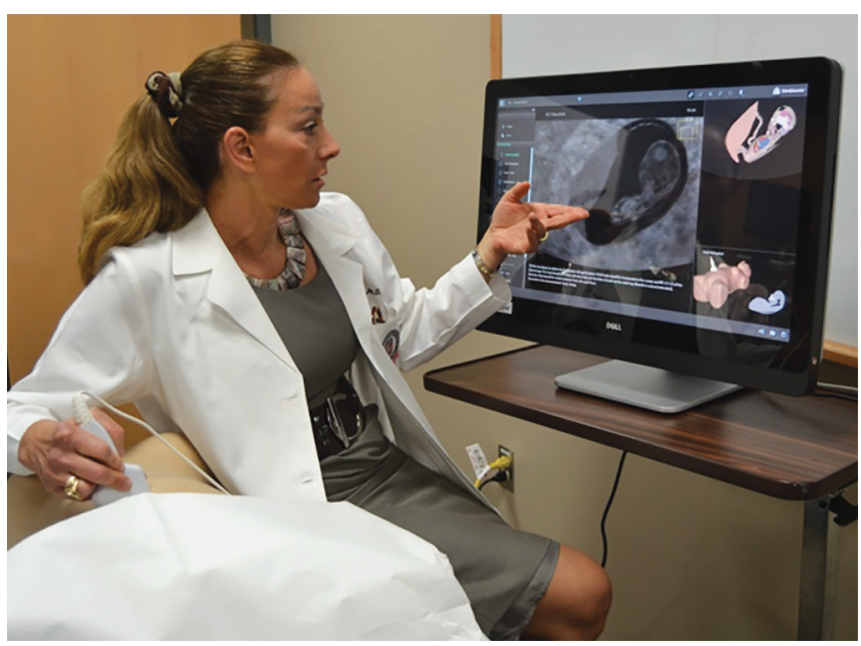

Fig. 2: Illustration of the first-trimester US evaluation and nuchal translucency measurement on U/S Mentor (3D Systems) performed by Dr Kupesic. Self-directed learning modules improve the scanning capabilities and clinical reasoning of her learners 
US examinations. The images are more realistic, but the probe's movements often do not correspond precisely to its real counterpart, and may miss some parts of the volume. ${ }^{8}$ Because the acquired planes are usually made in one direction, the orthogonal planes are reconstructed and therefore show poorer image quality and resolution.

4. Generative model-based simulation: 2D US images in generative model-based simulator reconstructed by the software. Image quality is largely dependent upon the angle applied between the mock probe and the phantom. ${ }^{11}$ Such models typically produce "cartoonlike" simplified images. ${ }^{12}$

\section{Advantages of US Simulation}

Ultrasound simulators provide learning opportunities in an unpressurised environment, which reduces the learner's stress and virtually excludes potential harm to the patients (enhanced patient safety). Recent study by Gibbs $^{13}$ evaluated different strategies for effective integration of simulation into US training and confirmed that its effectiveness was maximized when introduced in early stages, before interaction with real patients. Further research is needed to determine the role of US simulation in credentialing, revalidation, and identifying suboptimal performers.

\section{How to Adapt Simulation for Donald School Needs?}

Basic Donald School courses consist of the theoretical component (course attendance, Donald School textbooks and DVDs) and practical training. Based on regional branch needs assessment, each course director determines the minimum number of didactic sessions and US examinations each trainee should perform and report under supervision. A logbook listing of the type of examinations undertaken is kept for this purpose. Ultrasound training is supervised by someone who has previously obtained at least a basic and one or more advanced Donald School courses, typically a consultant level trainer (usually local and/or regional expert). During and following the completion of the US training course, based on the outcomes of the formative and summative competency assessment, the supervisor/proctor/instructor will determine in which area (or areas) the trainee can practice independently.

The Center for Advanced Teaching and Assessment in Clinical Simulation in El Paso, Texas, USA, accredited by the Society for Simulation in Health care for three standards teaching/education, assessment and research in clinical simulation conducted a three-year prospective, randomized, cohort trial evaluating the outcomes of pelvic US simulation course. ${ }^{14}$ Evident gain in knowledge and confidence following completion of the pelvic US simulation course confirmed that combination of simulation, standardized patient encounters, and one-on-one teaching by US experts have significantly improved US training experience. Postcourse survey indicated that all residents felt more comfortable in performing and interpreting transabdominal and transvaginal US in the simulated and real-world situations. ${ }^{14}$ According to trainees' feedback, one of the major advantages of the course was that board-certified physicians provided instruction in early stages of their training, so errors could be easily corrected and proper examination technique was mastered during each training session.

More recently, a virtual reality pelvic US simulation workshop using a new generation of simulation technology (VIMEDIX, CAE Health care, Sarasota, USA, and Ville St. Laurent, Quebec, CAN, and Simbionix US mentor, 3D Systems Health care, Littleton, CO, USA) was conducted at our simulation center. ${ }^{15}$ Pelvic US simulation activity lasted 4 hours and consisted of three modules: abnormal uterine bleeding, adnexal masses, and bleeding in pregnancy. All learners completed a pre- and postencounter quiz, and an anonymous postsimulation survey on relevance of US simulation to clinical learning and its usefulness to improve scanning performance and interpretation skills. About 31 participants attended the workshop, and $28(90.3 \%)$ of them responded to the survey. Five respondents agreed; 23 strongly agreed that pelvic US simulation applies to their clinical US practice; and 7 of them agreed and 21 strongly agreed that their performance and interpretation of US skills will be improved following their simulation training. The average postactivity knowledge score for all three topics significantly increased ( $\mathrm{p}$ value from a paired $t$-test; $p<0.0001)$. Postworkshop survey indicated that US simulation is a useful complement to learning on real patients that has a potential to improve their pelvic US performance, interpretation skills, and clinical reasoning. ${ }^{15}$

\section{CONCLUSION}

New generation of US simulators provides an opportunity for longitudinal monitoring of the learners' scanning skills based on the outcomes obtained by validated tools for assessment. Similar to other associations and/or societies, Ian Donald School is experiencing issues with establishing the metrics for US credentialing, looking for ways to insure sonographer/trainee competency and patient safety. ${ }^{16}$ The main advantages of US simulation are consistency, predictability, ability to customize, design and manipulate learning modules, practice rare cases, provide immediate and objective feedback, and implement clinical decision-making and critical thinking 
in a safe learning environment without compromising patient comfort and safety. Incorporation of simulation into an educational program provides unique opportunity for curriculum integration, repetitive practice, and individualized outcome-driven learning in a safe environment, ultimately decreasing training costs due to reduced working hours of US educators. In order to guarantee similar experience for its learners in different geographic locations, Ian Donald School should consider implementing US simulation for its educational and competency assessment activities. There is a need to develop a proficiency/competency-based US simulation for different level of learners in simulation training centers of excellence. These centers need to be appropriately equipped and staffed, ensuring experienced supervision and application of consistent metrics for formative and summative assessment.

Clearly, simulation cannot replace a valuable clinical experience, but can supplant a large part of basic skills training being performed on sick patients. We must remain faithful that simulation-based training has a potential to transform undergraduate, graduate, and continuous medical education by improving training and clinical outcomes. It is our obligation as educators of today to master the pedagogy of simulation-enhanced learning and serve as advocates and developers of the new simulation and virtual reality technology to train tomorrow's health care providers.

\section{REFERENCES}

1. Kobal SL, Trento L, Baharami S, Tolstrup K, Naqvi TZ, Cercek B, Neuman Y, Mirocha J, Kar S, Forrester JS, et al. Comparison of effectiveness of hand-carried ultrasound to bedside cardiovascular physical examination. Am J Cardiol 2005 Oct;96(7):1002-1006.

2. American Institute of Ultrasound in Medicine. AIUM practice guideline for the performance of obstetric ultrasound examinations. J Ultrasound Med 2013 Jun;32(6):1083-1101.

3. ISUOG Recommendations. ISUOG Education Committee recommendations for basic training in obstetric and gynecological ultrasound. Ultrasound Obstet Gynecol 2014 Jan;43(1):113-116.

4. ISUOG Education Committee. Update on proposed minimum standards for ultrasound training for residents in Ob/Gyn. Ultrasound Obstet Gynecol 1996 Nov;8(5):363-365.

5. Hertzberg BS, Kliewer MA, Bowie JD, Carroll BA, DeLong DH, Gray L, Nelson RC. Physician training requirements in sonography: How many cases are needed for competence? Am J Roentgenol 2000 May;174(5):1221-1227.

6. Gracias VH, Frankel H, Gupta R, Reilly PM, Gracias F, Klein W, Nisenbaum H, Schwab CW. The role of positive examinations in training for the focused assessment sonogram in trauma (FAST) examination. Am Surg 2002 Nov;68(11):1008-1011.

7. Kupesic Plavsic S. Distance learning at Ian Donald School. Donald School J Ultrasound Obstet Gynecol 2013 Jul-Sep;7(3): 51-62.

8. Chalouhi GE, Bernard V, Ville Y. Ultrasound simulators in obstetrics and gynecology: state of the art. Ultrasound Obstet Gynecol 2015 Sep;46(3):255-263.

9. Maul H, Scharf A, Baier P, Wustemann M, Gunter HH, Gebauer G, Sohn C. Ultrasound simulators: experience with the SonoTrainer and comparative review of other training systems. Ultrasound Obstet Gynecol 2004 Oct;24(5):581-585.

10. Madsen ME, Konge L, Nørgaard LN, Tabor A, Ringsted C, Klemmensen AK, Ottesen B, Tolsgaard MG. Assessment of performance measures and learning curves for use of a virtualreality ultrasound simulator in transvaginal ultrasound examination. Ultrasound Obstet Gynecol 2014 Dec;44(6):693-699.

11. Lewiss RE, Hoffmann B, Beaulieu Y, Phelan MB. Point-of-care ultrasound education: the increasing role of simulation and multimedia resources. J Ultrasound Med 2014 Jan;33(1):27-32.

12. Meller G. A typology of simulators for medical education. J Digit Imaging 1997 Aug;10(3 Suppl 1):194-196.

13. Gibbs V. The role of ultrasound simulators in educations: an investigation into sonography student experiences and clinical mentor perceptions. Ultrasound 2015 Nov;23(4):204-211.

14. Vallabh Patel, V, Mendez M, Kupesic Plavsic S. The importance of multimodality pelvic ultrasound simulation in teaching of OB GYN residents. Donald School J Ultrasound Obstet Gynecol 2014 Jan-Mar;8(1):1-5.

15. Arya S, Mulla ZD, Kupesic Plavsic S. Role of pelvic ultrasound simulation. The Clin Teacher 2017;14:1-5.

16. Kupesic Plavsic S. Strategies for teaching and assessing OB GYN ultrasound skills: Donald School model. Donald School J Ultrasound Obstet Gynecol 2014 Jan-Mar;8(1):11-15. 